Thomas Reuter*, Igor Ponomarev

\title{
Biphasic parameter identification of 3D scaffold-free cartilage transplants (SFCT) from stress relaxation compression tests using an optimized 3D FE-based method with tension-compression nonlinearity
}

\begin{abstract}
Cartilage constructs produced by SFCTtechnology provide promising opportunities to restore cartilage defects. Mechanical parameters of soft tissues are explicit markers for quantitative tissue characterization. In this study, we present a biphasic 3D-FE-based method to determine the biomechanical properties of SFCT from stress relaxation compression tests $(\varepsilon=20 \%, t=3400 \mathrm{~s})$ whereby cartilaginous tissue is modeled as a biphasic material with tension-compression nonlinearity (BMTCN). The FE-model computation was optimized by exploiting the axial symmetry and mesh resolution. The $\mathrm{R}^{2}$ of the fit results varies between 0.970 and 0.983 . The Young's and fiber modulus determined from SFCT are 37-times and 5-times lower than from native articular cartilage, respectively. Permeability, on the other hand, is 11-times higher than from native articular cartilage.
\end{abstract}

Keywords: Cartilage, Scaffold-free Cartilage Transplants (SFCT), Stress Relaxation, FE-modelling, Parameter Identification, Biphasic Theory, Tension-CompressionNonlinearity (TCN).

https://doi.org/10.1515/cdbme-2021-2090

\section{Introduction}

Articular cartilage and SFCT were investigated and characterized by biomechanical, biochemical and histological analysis. However, the in vitro mechanical stability of

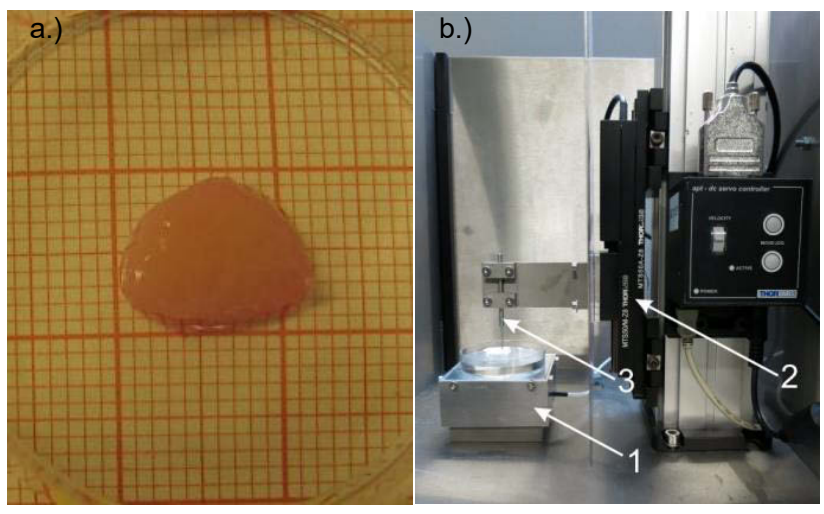

Figure 1:a.) Scaffold-free cartilage transplant and b.) Experimental setup. ( 1 force sensor, 2 linear stage, 3 compression plate)

cartilage constructs has not completely been investigated to date [1]. In particular, the biphas ic biomechanical properties reveal a variety of relevant information for the functional characterization of cartilaginous tis sue [2-4]. In this study, an optimized biphasic 3D-FE-model-based approach with TCN was implemented for an accurate description of the mechanical behaviour of SFCT. Material parameters (Young's modulus E, fiber modulus $\xi$ and permeability k) were extracted directly by fitting the numerical model to experimentally measured stress relaxation compression data $[2,5]$. Furthermore, the material parameters of SFCT and native articular cartilage were compared.

\section{Methods}

The determination of SFCT material properties was performed by a two-step approach [2]. First, stress relaxation compression measurements were conducted to record the resulting compression profile over time at a constant strain. Second, an optimized computational approach was developed
*Corresponding author: Thomas Re uter: ICM-Institut Chemnitzer Maschinen- und Anlagenbau e.V., Otto-SchmerbachStr. 19, 09117 Chemnitz, Germany, E-Mail: t.reuter@icmchemnitz.de

Igor Ponomarev: Research Centre of Medical Technique and Biotechnology, 99947 Bad Langensalza, Germany 
to identify the material properties of SFCT. This final step was performed by a FE-based parameter extraction model.

\subsection{SFCT Manufacturing and Cultivation}

The equine chondrocytes of the knee joint were used for the production of SFCT (cadaver specimens fromabattoir). After cultivation and proliferation in monolayer cultures, cells were trans formed into a three-dimensional structure without any artificial matrix, growth or differentiation factors (Fig. 1a) [6]. The cultivation of the SFCT was carried out in 6-well plates and medium was changed daily. The cultivation period was accompanied by the application of a cyclic manual mechanical loading. For the mechanical loading a plane glass stamp is used. The duration, frequency, and intensity of loading were determined under tactile and visual control [6]. SFCT $(n=5)$ were analyzed after 6 weeks of culturing and a 14-fold cyclic mechanical loading.

\subsection{Experimental Setup}

The unconfined compression tests were performed by a compression plate with a diameter of $20 \mathrm{~mm}$. The compression plate was placed perpendicular to the tissue surface. A custom-built stress relaxation-compression testing machine (fzmb GmbH, Bad Langensalza) was used to apply a strain of $\varepsilon=20 \%$ (SFCT) and $\varepsilon=10 \%$ (native articular cartilage) with $\mathrm{v}=0.02 \mathrm{~mm} / \mathrm{s}$ (Fig. 1b). The samples were mounted in a culture dish filled with PBS-solution. The resulting compression force was measured with a force sensor (ME-Systeme $($ ), Henningsdorf) placed under the culture dish. The following load profile was used: 1) Determination of the cartilage's surface (move compression plate until a load of $100 \mathrm{mN}$ is reached); 2) Recovery from the preload of step 1 for $10 \mathrm{~min}$; 3) Relaxation compression at $\varepsilon=20 \%$ and $\varepsilon=10 \%$ for $3400 \mathrm{~s}$ [2,7]. The SFCT are softer, therefore a higher strain was used. Measurements of cartilage thickness were taken as the difference between the surface of the culture dish and the cartilage surface. All tests were performed at $25^{\circ} \mathrm{C}$ in $\mathrm{PBS}$-solution.

\subsection{Native Articular Cartilage}

Equine medial and lateral anterior condyles were harvested from knee joints to carry out the stress relaxation compression experiments (cadaver specimens from abattoir, $\mathrm{n}=14$, age $5-14$ years, no visible damage, stored fresh frozen at $-20^{\circ} \mathrm{C}$ ).

\subsection{Computational Modelling}

For the calculation of the biomechanical parameters of cartilage / SFCT from stress relaxation compression, an FEbased parameter extraction modelwas applied. The software Finite Elements for Biomechanics (FEBio@), Version 2.3) was used to set up the FE-model and the parameter identification [8]. The model was configured in accordance with the experimental setup to match the individual dimensions andboundary conditions of each specimen. Due to the long processing time, a pseudo-axisymmetric wedge model was implemented (Fig. 2). Additionally, the FE-mesh resolution was adjusted to minimize the model error and to obtain mesh independent model results [9].

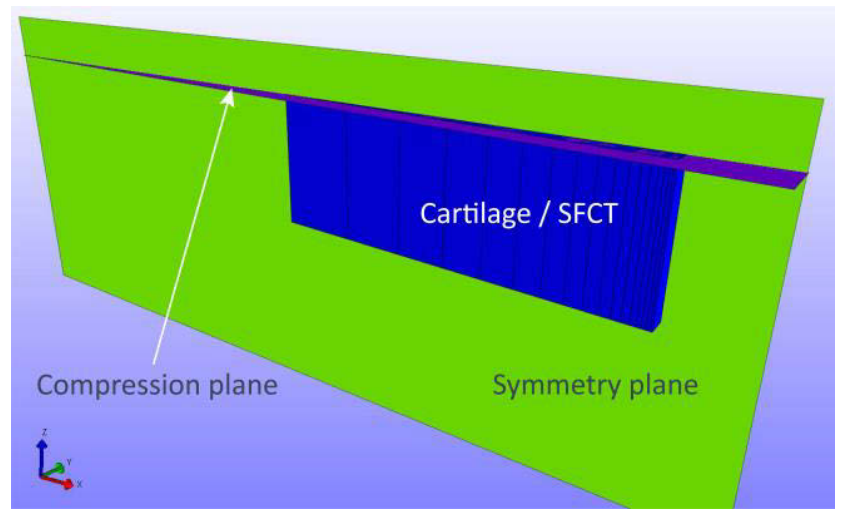

Figure 2: Axisymmetric FE-model, FE-mesh, compression plate position, and symmetry plane for the parameter identification routine.

An extended biphasic model was used for an accurate description of the mechanical behaviour of articular cartilage. According to the biphasic theory $[10,11]$, the tissue was assumed to consist of a compres sible solid matrix, hydrated with an incompressible fluid. The solid matrix is treated as a compressible isotropic neo-Hookean ground matrix reinforced with spherical fiber distribution. The fibers can only sustain tensile stress. Therefore, the compressive modulus of the material is defined to be the Young's modulus of the neo-Hookean ground matrix. The strain energy density function of the fiber bundles is defined as

$$
\psi=\frac{\xi}{\alpha \beta}\left(e^{\left[\alpha\left(I_{n}-1\right)^{\beta}\right]-1}\right)
$$

where $\xi$ is the fiber modulus, $\alpha$ and $\beta$ are constants and set to 0 and 2 to performan almost linear stress-strain relationship at small strains $[5,10]$. The material representation used consisted of a compressible is otropic neo-Hookean ground matrix reinforced with spherical fiber distribution properties, constant permeability and an inviscid fluid phase, which introduces four material parameters of Young's modulus, fiber modulus, Poisson's ratio and permeability of the solid 
matrix $[10,11]$. Poisson's ratio of the neo-Hookean ground matrix is assumed to be $0[2,5,12]$.

For the parameter identification, the parameter optimization module of FEBio(C) was used. This module iteratively identifies material parameters by solving an inverse finite element problem by coupling the FE-model to an optimization scheme that minimizes the error between the experimental results and the FE-predicted time-dependent stress relaxation curves. The Levenberg-Marquardt algorithm was used as optimization scheme $[8,12]$. The optimization objective function that had to be minimized was defined as the sum of squares of the difference between experimental and simulated stress relaxation curves. The coefficient of determination $\mathrm{R}^{2}$ of the optimization was calculated for the evaluation of the parameter identification [12].

\section{Results}

Experimental stress relaxation curves of five SFCT and one native articular cartilage represent typical viscoelastic relaxation behaviour of cartilage tissue (Fig. 4). However, SFCT show very low equilibrium force after $3400 \mathrm{~s}$ relaxation time compared to native cartilage. No meaningful changes occur in the measured force after about $t=1500 \mathrm{~s}$ (SFCT) and after about $\mathrm{t}=2100 \mathrm{~s}$ (native articular cartilage).

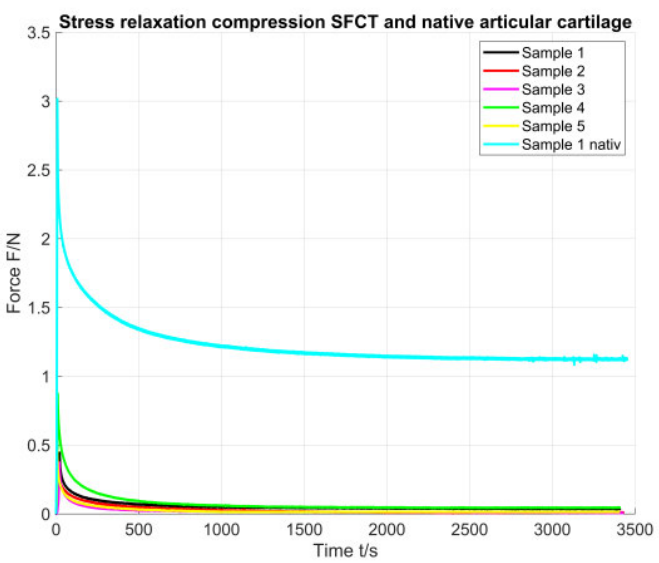

Figure 4: Experimental data fromstress relaxation compression tests.

The differences between simulation and experiment are small (e.g. sample no. 1, $\mathrm{R}^{2}=0.970$ (SFCT), Fig. 3). But the initial force peak is underestimated by the BMTCN-model. Overall, $\mathrm{R}^{2}$ varies between 0.970 and 0.983 (SFCT) and between 0.987 and 0.996 (native articular cartilage) for all the samples tested. The estimated material parameters of SFCT vary from 0.609 to $6.072 \mathrm{kPa}$ for Young's modulus E, 0.824 to $6.144 \mathrm{kPa}$ for fiber modulus $\xi$ and 0.220 to $1.719 \mathrm{~mm}^{4} / \mathrm{Ns}$ for permeability $\mathrm{k}$. The standard deviation of the SFCT is very high due to the individual manufacturing and cultivation process (Tab. 1).

Table 1: Material parameters calculated (SFCT).

\begin{tabular}{llll}
\hline Sample no. & $\begin{array}{l}\text { Young's } \\
\text { modulus } \\
\text { E [kPa] }\end{array}$ & $\begin{array}{l}\text { Fiber } \\
\text { modulus } \\
\xi[\mathrm{kPa}]\end{array}$ & $\begin{array}{l}\text { Perm eability } \\
\mathbf{k}\left[\mathrm{m} \mathrm{m}^{\mathbf{4}} / \mathrm{Ns}\right]\end{array}$ \\
\hline 1 & 2.217 & 0.977 & 1.512 \\
2 & 0.609 & 0.824 & 1.719 \\
3 & 2.477 & 1.787 & 1.466 \\
4 & 6.072 & 6.144 & 0.220 \\
5 & 2.597 & 3.480 & 0.273 \\
Mean \pm Std & $\mathbf{2 . 7 9 5 \pm 2 . 0 0 0}$ & $\mathbf{2 . 6 4 3} \pm \mathbf{2 . 2 2 4}$ & $\mathbf{1 . 0 3 8} \pm \mathbf{0 . 7 2 9}$ \\
\hline
\end{tabular}

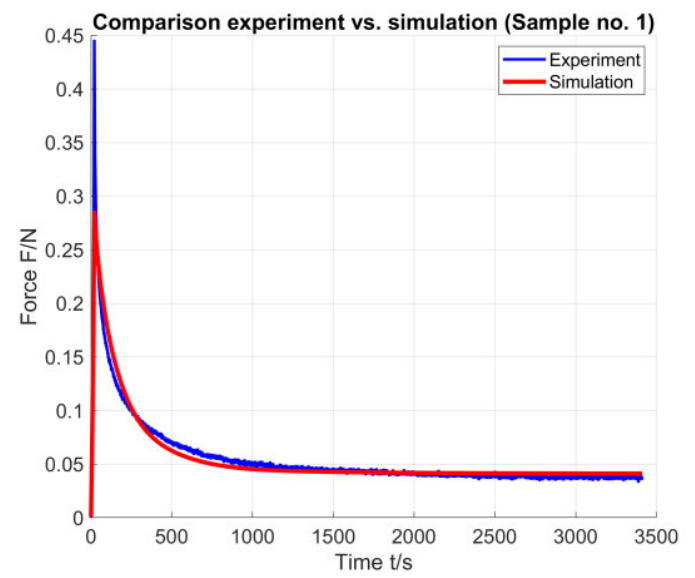

Figure 3: Comparison betw een simulation and experiment.

The results of the comparis on between SFCT and native equine articular cartilage (divided into medial and lateral condyles) show large significant differences (Fig. 5-7). The Young's and fiber modulus determined from SFCT are 37times and 5-times lower than from native articular cartilage, respectively. Permeability, on the other hand, is 11-times higher than fromnative articular cartilage. This indicates that SFCT is significantly softer than native cartilage.

\section{Conclusion}

The two-step method is a robust approach to extract the material parameters of SFCT. An optimized biphas ic 3D-FEbased method was utilized to characterize the mechanical behaviour of SFCT from stress relaxation compression data. The BMTCN-model described the experimental stress relaxation compression with high accuracy $\left(\mathrm{R}^{2}>0.97\right)$. But the initial stress relaxation behaviour cannot be correctly 
Biphas ic parameter identification of 3D s caffold-free cartilage transplants (SFCT) from stress relaxation compression tests using an optimized 3D FE-based method w ith tension-compression nonlinearity

reproduced by the BMTCN-model. Biomechanical differences between SFCT and native articular cartilage resulted probably from the incomplete maturing process of SFCT. As a result, SFCT are significantly softer than native articular cartilage. Further research is needed to develop an adequate material model for SFCT. Future work will focus on the characterization of the maturing process of SFCT.

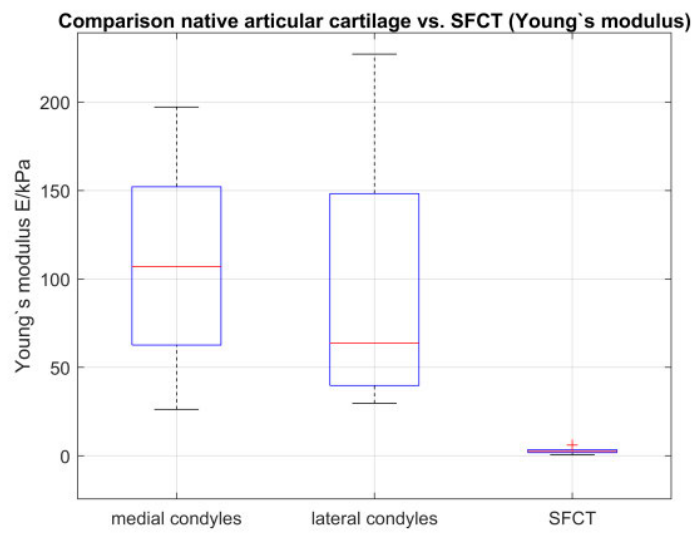

Figure 5: Comparison betw een native articular cartilage and SFCT (Young's modulus).

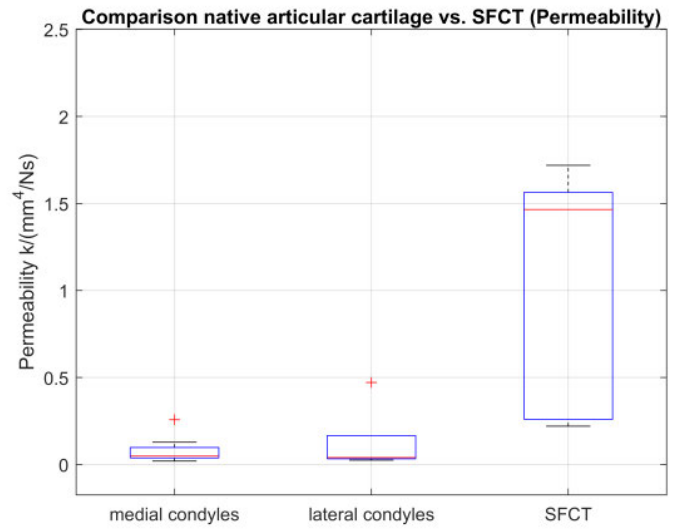

Figure 6: Comparison betw een native articular cartilage and SFCT (Permeability).

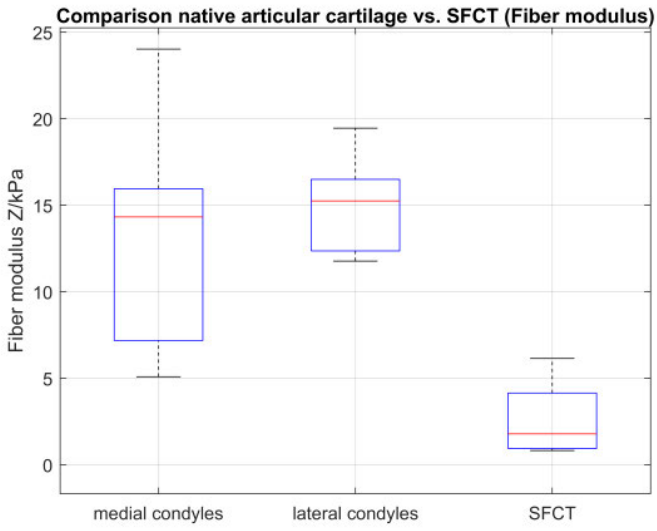

Figure 7: Comparison betw een native articular cartilage and SFCT (Fiber modulus).

\section{Author Statement}

Research funding: This research is supported by the German Ministry of Economy and Technology (INNO-KOM-OST Reg. Nr.: VF160052). Conflict of interest: Authors state no conflict of interest. Informed consent: Informed consent has been obtained from all individuals included in this study. Ethical approval: The conducted research is not related to human or animals use.

\section{References}

[1] Reuter T, Ponomarev I. Biomechanical parameter determination of scaffold-free cartilage constructs (SFCCs) $w$ ith the hyperelastic material models Yeoh, Ogden and Demiray. Current Directions in Biomedical Engineering. 2015; 1(1):442-445.

[2] Reuter T, Hurschler C. Biphasic parameter identification of equine articular cartilage from creep indentation data using an optimized 3D FE-based method. Current Directions in Biomedical Engineering. 2018; 4(1):481-484.

[3] Hasler EM, Herzog W, Wu JZ, Müller W, Wyss U. Articular cartilage biomechanics: theoretical models, material properties, and biosynthetic response. Crit Rev Biomed Eng. 1999; 27(6):415-88.

[4] Mow VC, Kuei SC, Lai WM, Armstrong CG. Biphasic creep and stress relaxation of articular cartilage in compression? Theory and experiments. J Biomech Eng. 1980 Feb; 102(1):73-84.

[5] Reuter T, Hurschler C. Comparison of biphasic material properties of equine articular cartilage fromstress relaxation indentation tests $w$ ith and $w$ ithout tension-compression nonlinearity. Current Directions in Biomedical Engineering. 2018; 4(1):485-488.

[6] Ponomarev N, Kochneva LM, Barnew itz D. Effect of 3D chondrocyte culturing conditions on the formation of extracellular matrix in cartilage tissue-engineering constructs. Bull Exp Biol Med. 2014 Feb; 156(4):548-55.

[7] Armstrong CG, Mow VC. The mechanical properties of articular cartilage. Bull Hosp Jt Dis Orthop Inst. 1983 Fall; 43(2):109-117.

[8] Maas SA, Ellis BJ, Ateshian GA, Weiss JA. FEBio: finite elements for biomechanics. J Biomech Eng. 2012 Jan; 134(1): 011005.

[9] Suh JK, Spliker RL. Indentation Analysis of Biphasic Articular Cartilage: Nonlinear Phenomena Under Finite Deformation. J Biomech Eng. 1994 Feb; 116:1-9.

[10] Chen X, Zhou Y, Wang L, Santare MH, Wan LQ, LuXL. Determining Tension-Compression Nonlinear Mechanical Properties of Articular Cartilage from Indentation Testing. Ann Biomed Eng. 2016 Apr; 44(4):1148-58.

[11] Mow VC, Ratcliffe A, Woo SLY. Biomechanics of Diarthrodial Jonits, Volume I, Part II, Chapter 15, S. 401-436, 1990.

[12] Reuter T. Determination of biomechanical parameters of articular cartilage $w$ ith the help of FEM using experimental stress relaxation and creep indentation data, ME, FSI Beuth University, Berlin, 2015. 